\title{
Plasma levels and expression of interleukin-37 in patients with immune thrombocytopenia
}

\author{
FENG ZHANG $^{1}$, XIAO-JUAN ZHU ${ }^{1}$, XIAO-JING ZHU ${ }^{2}$, YAN-XIA LIU ${ }^{1}$, \\ TING YUAN $^{1}$ and QING-MIN YAO ${ }^{1}$
}

${ }^{1}$ Department of Hematology, Shandong Provincial Hospital Affiliated to Shandong University, Jinan, Shandong 250021; ${ }^{2}$ Department of Orthopedics, Chinese Medicine Hospital of Linyi City, Linyi, Shandong 276003, P.R. China

Received September 4, 2018; Accepted May 23, 2019

DOI: $10.3892 /$ etm.2019.7824

\begin{abstract}
Interleukin (IL)-37 has an important role in autoimmune diseases by suppressing immunity and inflammation; however, the role of IL-37 in immune thrombocytopenia (ITP) has remained largely elusive. The present study aimed to investigate the expression of IL-37 and its potential role in the pathogenesis of ITP. The plasma levels and expression of IL-37 in the peripheral blood mononuclear cells of patients with active ITP, ITP patients in remission and healthy controls were measured by ELISA and reverse transcription-quantitative PCR, respectively. The levels of IL-37 in patients with ITP treated with and without glucocorticoids were also determined by ELISA. Specific anti-platelet glycoprotein (GP)IIb/IIIa and/or GPIb/IX autoantibodies were assayed by modified monoclonal antibody-specific immobilization of platelet antigens. The mean value of plasma IL-37 in ITP patients was slightly higher than that in healthy controls, but this was not statistically significant. There was no correlation between IL-37 and anti-platelet autoantibodies, and no significant difference in the IL-37 concentration was identified between patients treated with and without glucocorticoids. In addition, the correlation between IL-37 and the platelet count was analyzed, with no statistical significance observed. It was therefore concluded that IL-37 may not have a pivotal role in the development of ITP. However, the lack of significant differences may be due to the limited number of patients in different groups. A larger number of ITP patients should be enrolled in the future work and achieve more accurate results.
\end{abstract}

Correspondence to: Dr Qing-Min Yao, Department of Hematology, Shandong Provincial Hospital Affiliated to Shandong University, 324 Jingwu Road, Jinan, Shandong 250021, P.R. China E-mail: yaoqingmin1980@163.com

Key words: interleukin-37, immune thrombocytopenia, autoimmune disease, cytokine

\section{Introduction}

Immune thrombocytopenia (ITP) is an autoimmune disorder characterized by a low platelet count with mucocutaneous or other types of bleeding. The pathogenesis of ITP remains poorly understood, while immune disorders are thought to have an important implication. The presence of anti-platelet glycoprotein (GP) antibodies is also considered to be critically involved in ITP (1). In addition, a complex dysregulation of cellular mechanisms has been reported, including the imbalance of the type $1 \mathrm{~T}$-helper cell (Th1)/Th2 ratio (2), increased number of Th17 cells (3), decreased number or functional deficit of regulatory $\mathrm{T}$ cells $(4,5)$ and increased cytotoxic $\mathrm{T}$ lymphocyte-mediated cytotoxicity (6).

Interleukin (IL)-37, a novel anti-inflammatory cytokine previously known as interleukin-1 family member 7 before it was renamed, has a pivotal role in the suppression of immune responses (7-10). Alternative splicing of the human gene for IL-37 gives rise to 5 different isoforms: IL-37a, b, c, d and e, among which IL-37b is the most likely one to be biologically functional. IL-37 is widely expressed in several types of cells, tissues and organs, including peripheral blood mononuclear cells (PBMCs) (7). The major role of IL-37 is to decrease excessive inflammation in innate and adaptive immune diseases, mainly by inhibiting the expression, production and function of pro-inflammatory cytokines, including IL-1 $\alpha$, IL-6, tumor necrosis factor (TNF) and macrophage inflammatory protein-2. The abundance of these cytokines has been reported to increase with the silencing of endogenous IL-37 in human blood cells $(7,11)$. In vivo, IL-37-transgenic mice exhibited markedly reduced manifestations of endotoxemia, dextran sulphate sodium colitis, ischemia-reperfusion injury and obesity-induced inflammation (12-14). Furthermore, treatment with recombinant IL-37 in wild-type mice has been demonstrated to exert protective effects in several models of inflammation and injury (15-17).

Aberrant expression of IL-37 has been observed in several inflammatory and autoimmune diseases, including rheumatoid arthritis (RA) (18-21), systemic lupus erythematosus (SLE) $(22,23)$, inflammatory bowel disease (IBD) $(24,25)$, ankylosing spondylitis (AS) (26) and Graves' disease (GD) (27). However, the role of IL-37 in ITP has remained elusive. To 
investigate the expression of IL-37 and its potential role in the pathogenesis of ITP, the levels of IL-37 in ITP patients were measured and their correlation to disease activity was determined.

\section{Materials and methods}

Patients and controls. A total of 34 patients with ITP were enrolled in the present study (Table I), consisting of 18 newly diagnosed ITP patients with active disease (11 females and 7 males; age range, 19-62 years; median age, 39.89 years) and 16 patients in remission (10 females and 6 males; age range, 19-62 years; median age, 37.25 years). The platelet count of

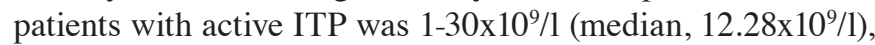
which was significantly lower than that of patients in remission $\left(101-305 \times 10^{9} / 1\right.$; median, $\left.163 \times 10^{9} / 1 ; \mathrm{P}<0.001\right)$. Out of the 34 patients with ITP, 12 were treated with glucocorticoids, including high-dose dexamethasone (HD-DEX) $40 \mathrm{mg}$ daily for 4 day severy 4 weeks and prednisone $1.0 \mathrm{mg} / \mathrm{kg}$ daily, which was then tapered.

The control group consisted of 15 healthy volunteers admitted to the hospital for routine physical examination (9 females and 6 males; age range, 16-66 years; median age, 39.67 years). The platelet count was $128-325 \times 10^{9} / 1$ (median, $\left.231 \times 10^{9} / 1\right)$.

The enrollment of patients and healthy volunteers took place between November 2016 and January 2018 at the Department of Hematology of the Shandong Provincial Hospital affiliated to Shandong University (Jinan, China). All cases met the diagnostic criteria of ITP, as previously described (28). Patients complicated with diabetes, hypertension, cardiovascular diseases, pregnancy, active or chronic infection or connective tissue diseases were excluded from the study.

PBMC preparation. Plasma samples were separated by centrifugation and stored at $-20^{\circ} \mathrm{C}$ to be used for the determination of IL-37 and anti-platelet autoantibodies.

PBMCs were isolated from heparinized blood samples by Ficoll-Hypaque density gradient centrifugation at $780 \mathrm{x}$ g for $20 \mathrm{~min}$ at $20^{\circ} \mathrm{C}$, and stored at $-80^{\circ} \mathrm{C}$ for future use.

ELISA of IL-37. The plasma concentration of IL-37 was measured by a commercial ELISA kit, according to the manufacturer's protocols (AdipoGen). The lower detection limit of this assay was $16 \mathrm{pg} / \mathrm{ml}$.

Determination of the mRNA expression of $I L-37$. For reverse transcription (RT), total RNA was extracted from PBMCs using TRIzol reagent (Thermo Fisher Scientific, Inc.). The RNA was then reverse transcribed to complementary DNA using the PrimeScript ${ }^{\mathrm{TM}}$ RT Reagent kit (Takara Bio, Inc.), according to the manufacturer's protocol. Quantitative (q)PCR for IL-37 was performed on an ABI PRISM_7500 Sequence Detection System (Thermo Fisher Scientific, Inc.) using SYBR Green (Toyobo Life Science) according to the manufacturer's protocol. $\beta$-Actin was used as the endogenous control. The sequences of specific primers were as follows: IL-37 forward, 5'-AAGACCTACGCCATGGGACATC-3' and reverse, 5'-TCT TGGTATTGCAAGTTGGAGTTCA-3'; $\beta$-actin forward, 5'-TTGCCGACAGGATGCAGAA-3' and reverse,5'-GCC
GATCCACACGGAGTACT-3'. The relative expression levels between IL-37 and $\beta$-actin were compared using the $2^{-\Delta \Delta C q}$ method (29).

Anti-platelet autoantibody determination. The specific anti-platelet autoantibodies to GPIIb/IIIa and/or GPIb/IX were analyzed by modified monoclonal antibody-specific immobilization of platelet antigens, as previously described (30).

Statistical analysis. Values are expressed as the mean \pm standard deviation. Statistical significance between two groups was determined using an unpaired Student's t-test. For multiple comparisons, one-way analysis of variance followed by Bonferroni's post-hoc test was used. The correlation analysis was performed using Pearson's correlation. All statistical analyses were performed using SPSS 13.0 (SPSS, Inc.). P<0.05 was considered to indicate a statistically significant difference.

\section{Results}

IL-37 expression in ITP patients and controls. Fig. 1A presents the plasma concentration of IL-37 in the ITP and control groups. The mean plasma level of IL-37 in the ITP patients $(90.41 \pm 32.56 \mathrm{pg} / \mathrm{ml})$ was higher than that in normal controls $(75.62 \pm 27.52 \mathrm{pg} / \mathrm{ml})$, but this was not statistically significant (P>0.05). In Fig. 1B, the mRNA levels of IL-37 in PBMCs determined using the $2^{-\triangle \Delta C q}$ method are presented as the fold change in gene expression normalized to an endogenous reference gene ( $\beta$-actin) and relative to normal controls. The relative mRNA expression of IL-37 in untreated patients was 1.07 times that in the normal controls, with no statistically significant difference observed ( $\mathrm{P}>0.05$; Fig. 1B).

IL-37 expression in patients with active ITP and in remission. To investigate any potential correlation between IL-37 and disease activity, the concentration of plasma IL-37 and IL-37 mRNA were further analyzed in patients with active ITP, patients in remission and healthy controls. The concentration of plasma IL-37 in the different groups was as follows: $98.75 \pm 36.85 \mathrm{pg} / \mathrm{ml}$ (active ITP patients), $81.04 \pm 24.83 \mathrm{pg} / \mathrm{ml}$ (ITP patients in remission) and $75.62 \pm 27.52 \mathrm{pg} / \mathrm{ml}$ (normal controls); no significant differences were observed among active ITP patients, patients in remission and healthy controls ( $\mathrm{P}>0.05$; Fig. 2A). Despite not reaching statistical significance, the P-value for the comparison of plasma levels of IL-37 between active ITP patients and controls was 0.107, as determined by Bonferroni's post-hoc test. Similarly, no significant difference in the IL-37 mRNA expression was observed among the three groups ( $\mathrm{P}>0.05$; Fig. $2 \mathrm{~B})$.

Plasma IL-37 concentration in anti-platelet autoantibody positive/negative patients. Since anti-platelet autoantibodies have an important role in the pathogenesis of ITP, the concentration of IL-37 was further compared in anti-platelet autoantibody-positive and -negative patients. Fig. 3A presents the concentration of IL-37 in anti-GPIIb/IIIa-positive and -negative ITP patients. No significant difference was identified between anti-GPIIb/IIIa-positive $(83.06 \pm 22.02 \mathrm{pg} / \mathrm{ml})$ and -negative ITP patients $(93.93 \pm 36.47 \mathrm{pg} / \mathrm{ml} ; \mathrm{P}>0.05)$, or in 
Table I. Clinical characteristics of ITP patients.

\begin{tabular}{|c|c|c|c|c|c|}
\hline Case no. & Sex/age (years) & Plt count $\left(\mathrm{x} 10^{9} / 1\right)$ & $\begin{array}{l}\text { Anti-GPIIb/IIIa/ } \\
\text { anti-GPIb/IX }\end{array}$ & Bleeding symptoms & Medications \\
\hline 1 & $\mathrm{~F} / 38$ & 5 & $-/-$ & PT & NONE \\
\hline 2 & $\mathrm{~F} / 25$ & 12 & $+/+$ & PT & NONE \\
\hline 3 & $\mathrm{M} / 48$ & 9 & $+/-$ & $\mathrm{EC}, \mathrm{GH}$ & NONE \\
\hline 4 & $\mathrm{~F} / 27$ & 18 & $-/-$ & PT, EC & NONE \\
\hline 5 & $\mathrm{~F} / 24$ & 10 & $-/-$ & PT & NONE \\
\hline 6 & $\mathrm{~F} / 35$ & 21 & $-/+$ & $\mathrm{EC}$ & NONE \\
\hline 7 & $\mathrm{M} / 23$ & 3 & $-/+$ & PT, EC & NONE \\
\hline 8 & $\mathrm{M} / 29$ & 15 & $-/-$ & PT, GH & NONE \\
\hline 9 & $\mathrm{~F} / 38$ & 30 & $+/+$ & $\mathrm{EC}$ & NONE \\
\hline 10 & $\mathrm{~F} / 59$ & 13 & $+/-$ & PT, GUH & NONE \\
\hline 11 & $\mathrm{M} / 42$ & 4 & $-/+$ & $\mathrm{EC}$ & NONE \\
\hline 12 & $\mathrm{~F} / 62$ & 1 & $-/-$ & PT, EC, GUH & Plt \\
\hline 13 & $\mathrm{~F} / 58$ & 17 & $-/+$ & PT, EC & NONE \\
\hline 14 & $\mathrm{M} / 47$ & 2 & $+/+$ & PT, GH & NONE \\
\hline 15 & $\mathrm{~F} / 37$ & 8 & $-/+$ & PT, EP & NONE \\
\hline 16 & $\mathrm{~F} / 48$ & 11 & $-/-$ & PT & NONE \\
\hline 17 & $\mathrm{M} / 59$ & 19 & $+/-$ & GUH & NONE \\
\hline 18 & $\mathrm{M} / 19$ & 23 & $-/-$ & PT & NONE \\
\hline 19 & $\mathrm{~F} / 49$ & 162 & $-/-$ & NONE & Pred, R \\
\hline 20 & $\mathrm{M} / 26$ & 104 & $-/-$ & NONE & IVIG, Plt \\
\hline 21 & $F / 29$ & 203 & $+/+$ & NONE & HD-DEX \\
\hline 22 & $\mathrm{~F} / 62$ & 113 & $-/-$ & NONE & Plt, R \\
\hline 23 & $\mathrm{M} / 50$ & 134 & $+/-$ & NONE & Plt, HD-DEX \\
\hline 24 & $\mathrm{~F} / 48$ & 224 & $-/-$ & NONE & IVIG, HD-DEX \\
\hline 25 & $\mathrm{~F} / 25$ & 180 & $+/-$ & NONE & HD-DEX \\
\hline 26 & $\mathrm{~F} / 31$ & 158 & $-/+$ & NONE & HD-DEX \\
\hline 27 & $\mathrm{M} / 49$ & 123 & $-/-$ & NONE & Pred, HD-DEX, R \\
\hline 28 & $\mathrm{~F} / 24$ & 305 & $-/-$ & NONE & HD-DEX \\
\hline 29 & $\mathrm{M} / 55$ & 150 & $+/-$ & NONE & Plt, R \\
\hline 30 & $\mathrm{~F} / 29$ & 107 & $-/-$ & NONE & IVIG, HD-DEX \\
\hline 31 & $\mathrm{~F} / 51$ & 158 & $+/-$ & NONE & HD-DEX,R \\
\hline 32 & $\mathrm{M} / 23$ & 137 & $-/-$ & NONE & HD-DEX \\
\hline 33 & $\mathrm{~F} / 19$ & 101 & $-/-$ & NONE & Plt, R \\
\hline 34 & $\mathrm{M} / 26$ & 249 & $-/-$ & NONE & IVIG, HD-DEX \\
\hline
\end{tabular}

Groups: Cases no. 1-18, ITP patients with active disease; cases no. 19-34, patients in remission. M, male; F, female; EC, ecchymoses; EP, epistaxis; GH, gingival hemorrhage; GUH, genitourinary hemorrhage; PT, petechiae; HD-DEX, high-dose dexamethasone; IVIG, intravenous gamma globulin; Plt, platelet (infusion); Pred, prednisone; R, rituximab; GP, glycoprotein.

the plasma levels of IL-37 between anti-GPIIb/IIIa-positive and -negative ITP patients, as compared with that in normal controls (75.62 $\pm 27.52 \mathrm{pg} / \mathrm{ml} ; \mathrm{P}>0.05)$. Similar results were obtained for anti-GPIb/IX-positive/negative ITP patients (P>0.05; Fig. 3B).

Influence of glucocorticoids on plasma IL-37 concentration in ITP patients. Glucocorticoids are used as a first-line therapy for ITP. In order to investigate any potential difference between patients treated with and without glucocorticoids, the concentration of plasma IL-37 was analyzed in different groups. The concentration of IL-37 was as follows: ITP patients treated without glucocorticoids $(97.06 \pm 36.45 \mathrm{pg} / \mathrm{ml})$, ITP patients treated with glucocorticoids $(78.23 \pm 19.84 \mathrm{pg} / \mathrm{ml})$ and normal controls $(75.62 \pm 27.52 \mathrm{pg} / \mathrm{ml})$; no significant difference was observed ( $\mathrm{P}>0.05$; Fig. 4$)$.

Correlation between IL-37 and platelet count in ITP patients. The correlation between plasma levels of IL-37 and platelet count in patients with active ITP was assessed by Pearson's correlation analysis and no significant correlation was identified $(\mathrm{P}>0.05)$. 

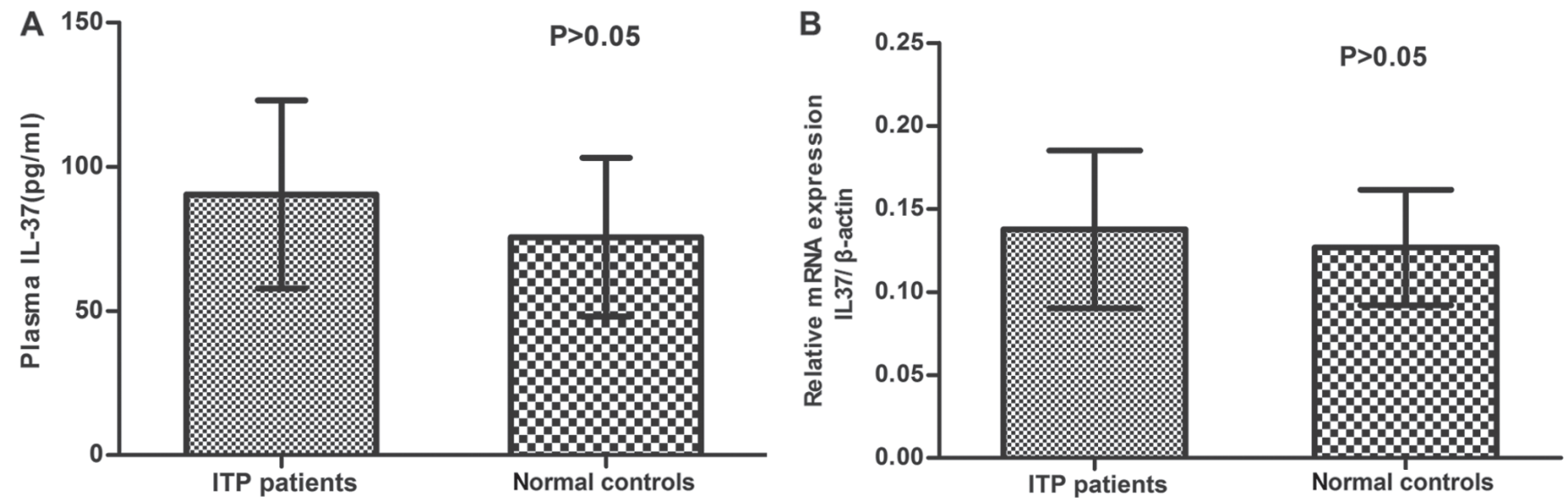

Figure 1. IL-37 levels in ITP patients and controls. (A) Analysis of plasma IL-37 concentrationin 34 ITP patients and 15 normal controls. No significant difference was observed between ITP patients and controls $(\mathrm{P}>0.05)$. (B) No significant difference in IL-37 mRNA expression in peripheral blood mononuclear cells was observed between ITP patients and controls $(\mathrm{P}>0.05)$. IL, interleukin; ITP, immune thrombocytopenia.
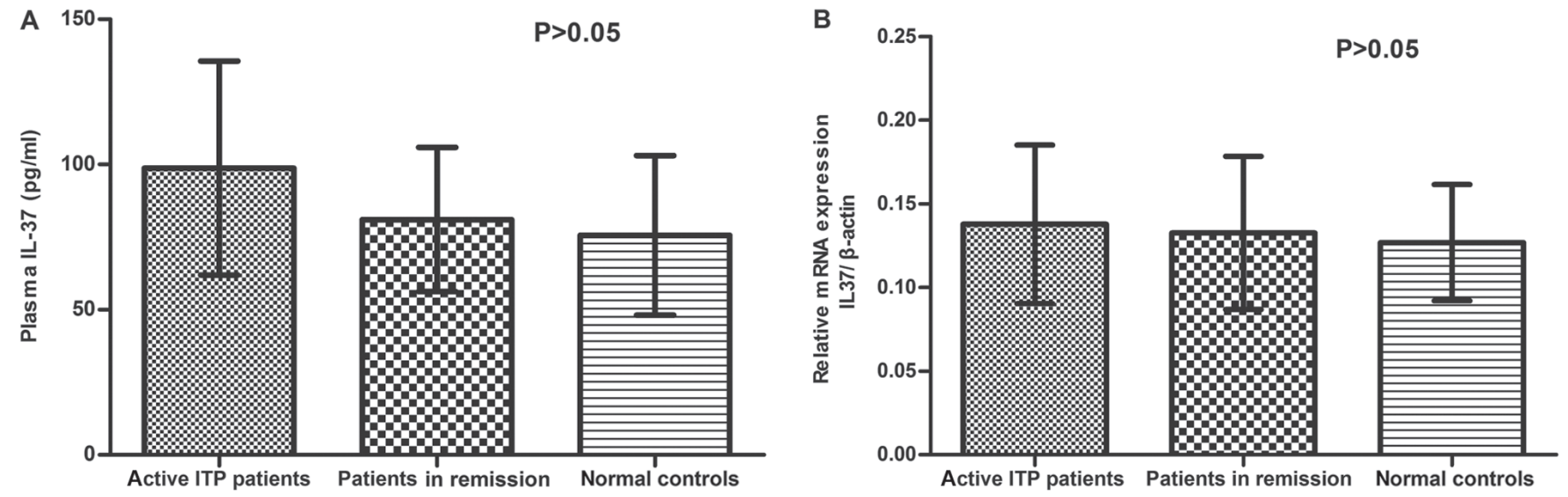

Figure 2. IL-37 levels in patients with active ITP, patients in remission and controls. (A) Plasma IL-37 concentration and (B) IL-37 mRNA expression in peripheral blood mononuclear cells of patients with active ITP, patients in remission and normal controls. No significant difference was observed among groups (P>0.05). IL, interleukin; ITP, immune thrombocytopenia.
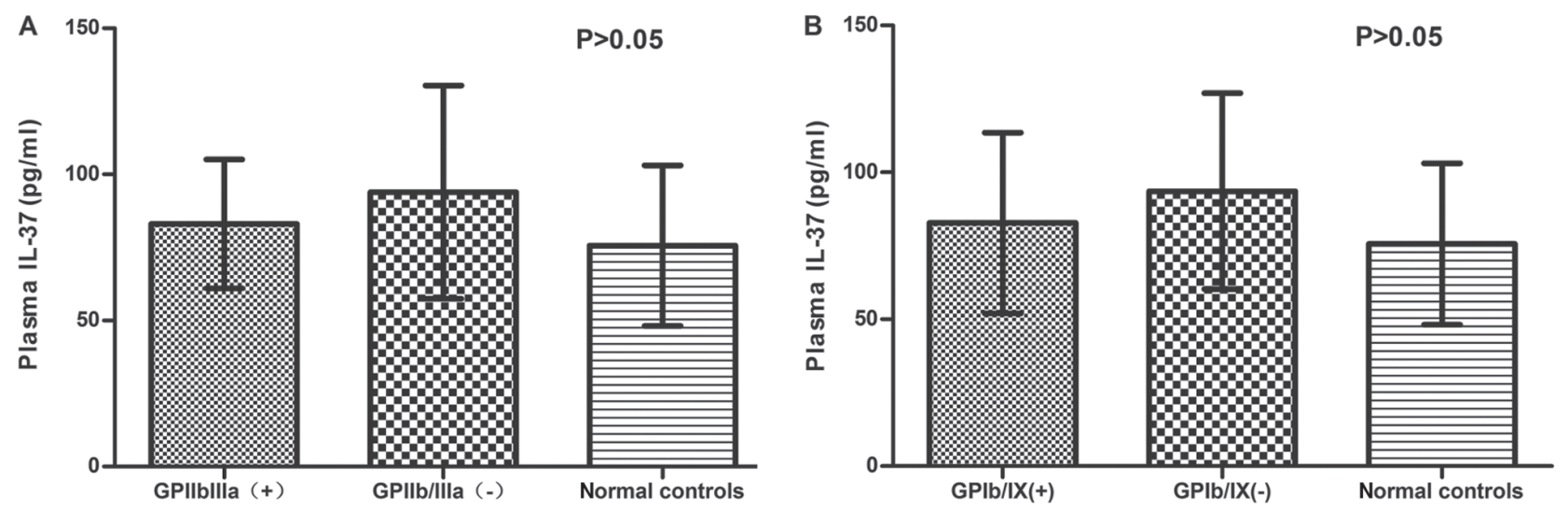

Figure 3. Plasma IL-37 concentration in anti-platelet autoantibody positive/negative patients. Analysis of plasma IL-37 from (A) anti-GPIIb/IIIa and (B) anti-GPIb/IX positive/negative ITP patients and controls. No significant difference was identified among the groups (P $>0.05)$. IL, interleukin; ITP, immune thrombocytopenia; GP, glycoprotein.

\section{Discussion}

Autoimmune diseases, including ITP, are characterized by impaired function caused by an immune response, in which abnormal antibodies are produced and attack the body itself. IL-37, a novel member of the IL-1 family, has been recognized as an important anti-inflammatory cytokine expressed by immune cells. Abnormal expression of IL-37 has been reported 


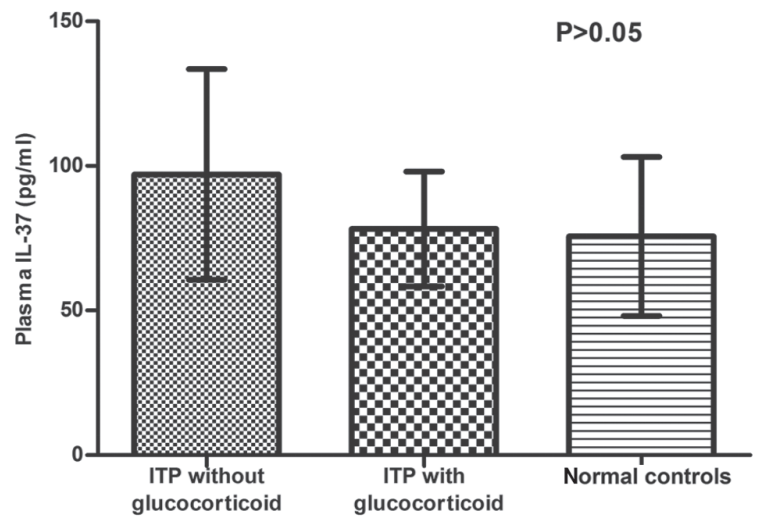

Figure 4. Plasma IL-37 concentration in ITP patients treated with and without glucocorticoids. Levels of plasma IL-37 were analyzed indifferent ITP groups: Patients treated with glucocorticoids, patients treated without glucocorticoids and normal controls. No significant difference was observed $(\mathrm{P}>0.05)$. IL, interleukin; ITP, immune thrombocytopenia.

in various autoimmune diseases, including SLE, RA, IBD, GD and AS (18-27), as a potential negative factor influencing the development of these disorders. According to Ye et al (22), increased IL-37 expression was associated with SLE disease activity, and IL-37 levels were significantly higher in patients with renal disease. Xia et al (20) indicated that the expression of IL-37 was markedly increased in RA patients, and a significant correlation was identified between IL-37 levels and disease activity. In addition, patients with active AS and GD had higher levels of IL-37 than those with inactive AS and GD and healthy controls $(26,27)$. All aforementioned data suggest that the expression of IL-37 is associated with disease activity of the above autoimmune diseases. Furthermore, inflammatory cytokine expression was higher in patients with active disease, as compared with that in patients with inactive disease and healthy controls. Functional analysis indicated that certain pro-inflammatory cytokines, including IL-1/6/10 and TNF- $\alpha$, may be involved in promoting the expression of IL-37, while high IL-37 expression may inhibit the overproduction of pro-inflammatory cytokines in autoimmune diseases through a negative feedback mechanism (31).

While most studies have reported an increased IL-37 expression in autoimmune diseases, decreased IL-37 expression was also identified in other autoimmune conditions, including Behcet's disease (32), asthma (33), Vogt-Koyanagi-Harada disease (34) and allergic rhinitis (35). The inconsistent expression of IL-37 among different autoimmune diseases may be due to differences in their immunological mechanisms. As mentioned above, IL-37 levels frequently exhibit a positive correlation with disease activity. This means that infection or inflammation increases IL-37 expression, while a high level of IL-37 may help to reduce the disease severity through a negative feedback mechanism. Conversely, low levels of IL-37 may indicate more severe inflammation.

To date, the role of IL-37 in ITP patients has remained elusive. In the present study, the plasma concentration of IL-37 and its mRNA expression in PBMCs of ITP patients was determined for the first time by using ELISA and RT-qPCR, respectively. The results indicated no significant difference in IL-37 levels between ITP patients and controls. In addition,

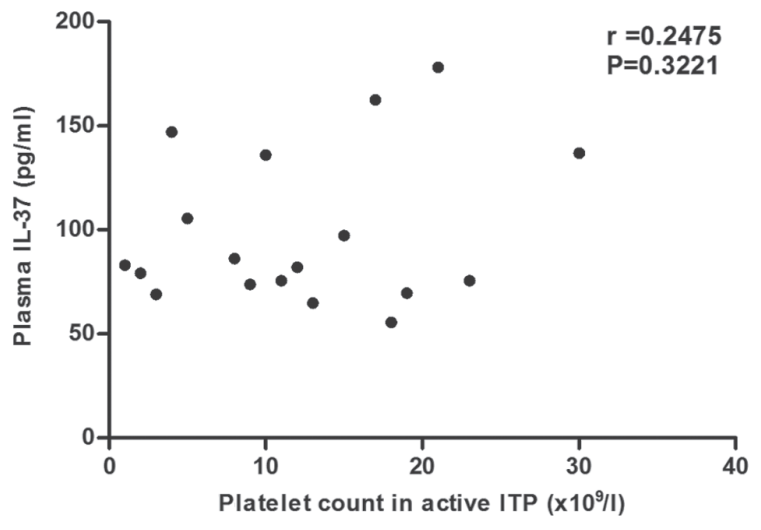

Figure 5. Correlation between IL-37 and platelet count. The correlation between plasma IL-37 and platelet count in active ITP patients was assessed by Pearson's correlation analysis, and no significant correlation was identified $(\mathrm{r}=0.2475, \mathrm{P}>0.05)$. IL, interleukin; ITP, immune thrombocytopenia.

no correlation was identified between IL-37 and anti-platelet autoantibodies. The correlation between IL-37 and the platelet count was also analyzed, with no statistical significance observed. However, the mean value of plasma IL-37 in ITP, particularly active ITP, was much higher than that in the controls. However, the P-value for the comparison of plasma IL-37 between patients with active ITP and controls was 0.107, as determined by Bonferroni's post-hoc test. Further studies with a bigger sample size are required to confirm these results, which may obtain a higher statistical significance.

It has been reported that IL-37 is able to translocate into the nucleus and downregulate pro-inflammatory cytokines (36), suggesting that IL-37 may also have an intracellular, in addition to its extracellular, function. Further studies examining the expression of intracellular IL-37 by methods including flow cytometry may be required.

Glucocorticoids, including conventional prednisone and HD-DEX, have been recommended as first-line therapy for ITP patients. Song et al (23) reported that glucocorticoids are able to downregulate the increased expression of IL-37 in SLE. The concentration of IL-37 was analyzed in ITP patients treated with and without glucocorticoids, with no significant difference observed between the two groups, which indicates that glucocorticoids may not regulate IL-37 in ITP patients. There are several explanations for this. First, the patients included in the present study were treated with glucocorticoids days, months or years ago, and thus, the glucocorticoid medication or blood sample collection time was inconsistent. Furthermore, the patients treated with or without glucocorticoids were not the same patients, and in a future study, assessment of the concentration of IL-37 in the same patients prior to and after glucocorticoid treatment may be more meaningful.

Accumulating evidence suggests that IL-37 has a pivotal role in autoimmune diseases. In the present study, the expression of IL-37 in ITP patients was evaluated for the first time, but no significantly abnormal expression of IL-37 was identified in these patients. It was therefore concluded that IL-37 may not have a pivotal role in the development of ITP. However, the lack of significant differences may be due to the limited 
number of patients in different groups. Larger number of ITP patients should be enrolled in the future work and achieve more accurate results.

\section{Acknowledgements}

Not applicable.

\section{Funding}

The present study was supported by a grant from the National Natural Science Foundation of China (grant no. 81100335).

\section{Availability of data and materials}

All data generated or analyzed during the present study are included in this published article.

\section{Authors' contributions}

FZ contributed to experimental design, data analysis and manuscript writing; X-JuZ contributed to case collection, literature search and manuscript writing; $\mathrm{X}-\mathrm{JiZ}$ and TY performed the experiments and statistical analysis; YL contributed to case collection, data interpretation and figure creation; QY was responsible for data interpretation, literature search, paper revision and submission.

\section{Ethics approval and consent to participate}

The study was approved by the Medical Ethics Committee of Shandong Provincial Hospital affiliated to Shandong University (Jinan, China). Written informed consent was obtained from all patients and/or their guardians.

\section{Patient consent for publication}

Not applicable.

\section{Competing interests}

The authors declare no competing interests.

\section{References}

1. Stasi R, Evangelista ML, Stipa E, Buccisano F, Venditti A and Amadori S: Idiopathic thrombocytopenic purpura: Current concepts in pathophysiology and management. Thromb Haemost 99: 4-13, 2008.

2. Panitsas FP, Theodoropoulou M, Kouraklis A, Karakantza M, Theodorou GL, Zoumbos NC, Maniatis A and Mouzaki A: Adult chronic idiopathic thrombocytopenic purpura (ITP) is the manifestation of a type-1 polarized immune response. Blood 103 . 2645-2647, 2004.

3. Zhang J, Ma D, Zhu X, Qu X, Ji C and Hou M: Elevated profile of Th17, Th1 and Tc1 cells in patients with immune thrombocytopenic purpura. Haematologica 94: 1326-1329, 2009.

4. Liu B, Zhao H, Poon MC, Han Z, Gu D, Xu M, Jia H, Yang R and Han ZC: Abnormality of CD4(+)CD25(+) regulatory T cell in idiopathic thrombocytopenic purpura. Eur J Haematol 78 139-143, 2007.

5. Yu J, Heck S, Patel V, Levan J, Yu Y, Bussel JB and Yazdanbakhsh K: Defective circulating CD25 regulatory T cells in patients with chronic immune thrombocytopenic purpura. Blood 112: 1325-1328, 2008
6. Zhao C, Li X, Zhang F, Wang L, Peng J and Hou M: Increased cytotoxic T-lymphocyte-mediated cytotoxicity predominant in patients with idiopathic thrombocytopenic purpura without platelet autoantibodies. Haematologica 93: 1428-1430, 2008.

7. Nold MF, Nold-Petry CA, Zepp JA, Palmer BE, Bufler P and Dinarello CA: IL-37 is a fundamental inhibitor of innate immunity. Nat Immunol 11: 1014-1022, 2010.

8. Luo Y, Cai X, Liu S, Wang S, Nold-Petry CA, Nold MF, Bufler P, Norris D, Dinarello CA and Fujita M: Suppression of antigen-specific adaptive immunity by IL-37 via induction of tolerogenic dendritic cells. Proc Natl Acad Sci USA 111: 15178-15183, 2014

9. Dinarallo CA and Bufler P: Interleukin-37. Semin Immunol 25: 466-468, 2013.

10. Bufler P,Gamboni-Robertson F,Azam T,Kim SH and Dinarello CA: Interleukin-1 homologues IL-1F7b and IL-18 contain functional mRNA instability elements within the coding region responsive to lipopolysaccharide. Biochem J 381: 503-510, 2004.

11. Gao W, Kumar S, Lotze MT, Hanning C, Robbins PD and Gambotto A: Innate immunity mediated by the cytokine IL-1 homologue 4 (IL-1H4/IL-1F7) induces IL-12-dependent adaptive and profound antitumor immunity. J Immunol 170: 107-113, 2003.

12. McNamee EN, Masterson JC, Jedlicka P, McManus M, Grenz A, Collins CB, Nold MF, Nold-Petry C, Bufler P, Dinarello CA and Rivera-Nieves J: Interleukin 37 expression protects mice from colitis. Proc Natl Acad Sci USA 108: 16711-16716, 2011.

13. Sakai N, Van Sweringen HL, Belizaire RM, Quillin RC, Schuster R, Blanchard J, Burns JM, Tevar AD, Edwards MJ and Lentsch AB: Interleukin-37 reduces liver inflammatory injury via effects on hepatocytes and non-parenchymal cells. J Gastroenterol Hepatol 27: 1609-1616, 2012.

14. Ballak DB, van Diepen JA, Moschen AR, Jansen HJ, Hijmans A, Groenhof GJ, Leenders F, Bufler P, Boekschoten MV, Müller M, et al: IL-37 protects against obesity-induced inflammation and insulin resistance. Nat Commun 5: 4711, 2014.

15. Coll-Miro M, Francos-Quijorna I, Santos-Nogueira E, Torres-Espin A, Bufler P, Dinarello CA and López-Vales R: Beneficial effects of IL-37 after spinal cord injury in mice. Proc Natl Acad Sci USA 113: 1411-1416, 2016.

16. Li S, Neff CP, Barber K, Hong J, Luo Y, Azam T, Palmer BE, Fujita M, Garlanda C, Mantovani A, et al: Extracellular forms of IL-37 inhibit innate inflammation in vitro and in vivo but require the IL-1 family decoy receptor IL-1R8. Proc Natl Acad Sci USA 112: 2497-2502, 2015.

17. Lunding L, Webering S, Vock C, Schröder A, Raedler D, Schaub B, Fehrenbach $\mathrm{H}$ and Wegmann M: IL-37 requires IL-18R $\alpha$ and SIGIRR/IL-1R8 to diminish allergic airway inflammation in mice. Allergy 70: 366-373, 2015.

18. Yang L, Zhang J, Tao J and Lu T: Elevated serum levels of Interleukin-37 are associated with inflammatory cytokines and disease activity in rheumatoid arthritis. APMIS 123: 1025-1031, 2015.

19. Xia L, Shen H and Lu J: Elevated serum and synovial fluid levels of interleukin-37 in patients with rheumatoid arthritis: Attenuated the production of inflammatory cytokines. Cytokine 76: 553-557, 2015.

20. Xia T, Zheng XF, Qian BH, Fang H, Wang JJ, Zhang LL, Pang YF, Zhang J, Wei XQ, Xia ZF and Zhao DB: Plasma interleukin-37 is elevated in patients with rheumatoid arthritis: Its correlation with disease activity and Th1/Th2/Th17-related cytokines. Dis Markers 2015: 795043, 2015.

21. Zhao PW, Jiang WG, Wang L, Jiang ZY, Shan YX and Jiang YF: Plasma levels of IL-37 and correlation with TNF- $\alpha$, IL-17A, and disease activity during DMARD treatment of rheumatoid arthritis. PLoS One 9: e95346, 2014.

22. Ye L, Ji L, Wen Z, Zhou Y, Hu D, Li Y, Yu T, Chen B, Zhang J, Ding L, et al: IL-37 inhibits the production of inflammatory cytokines in peripheral blood mononuclear cells of patients with systemic lupus erythematosus: Its correlation with disease activity. J Transl Med 12: 69, 2014

23. Song L, Qiu F, Fan Y, Ding F, Liu H, Shu Q, Liu W and Li X: Glucocorticoid regulates interleukin-37 in systemic lupus erythematosus. J Clin Immunol 33: 111-117, 2013.

24. Farrokhi M, Rezaei A, Amani-Beni A, Etemadifar M, Kouchaki E and Zahedi A: Increased serum level of IL-37 in patients with multiple sclerosis and neuromyelitis optica. Acta Neurol Belg 115: 609-614, 2015.

25. Imaeda H, Takahashi K, Fujimoto T, Kasumi E, Ban H, Bamba S, Sonoda H, Shimizu T, Fujiyama Y and Andoh A: Epithelial expression of interleukin-37b in inflammatory bowel disease. Clin Exp Immunol 172: 410-416, 2013. 
26. Chen B, Huang K, Ye L, Li Y, Zhang J, Zhang J, Fan X, Liu X, $\mathrm{Li} \mathrm{L}$, Sun J, et al: Interleukin-37 is increased in ankylosing spondylitis patients and associated with disease activity. J Trans Med 13: 36, 2015.

27. Li Y, Wang Z, Yu T, Chen B, Zhang J, Huang $\mathrm{K}$ and Huang Z: Increased expression of IL-37 in patients with Graves' disease and its contribution to suppression of proinflammatory cytokines production in peripheral blood mononuclear cells. PLoS One 9: e107183, 2014.

28. British Committee for Standards in Haematology General Haematology Task Force: Guidelines for the investigation and management of idiopathic thrombocytopenic purpura in adults children and in pregnancy. Br J Haematol 120: 574-596, 2003.

29. Livak KJ and Schmittgen TD: Analysis of relative gene expression data using real-time quantitative PCR and the 2(-Delta Delta C(T)) method. Methods 25: 402-408, 2001.

30. Hou M, Peng J, Shi Y, Zhang C, Qin P, Zhao C, Ji X, Wang X and Zhang M: Mycophenolatemofetil (MMF) for the treatment of steroid-resistant idiopathic thrombocytopenic purpura. Eur J Haematol 70: 353-357, 2003.
31. Xu WD, Zhao Y and Liu Y: Insights into IL-37, the role in autoimmune diseases. Autoimmun Rev 14: 1170-1175, 2015.

32. Bouali E, Kaabachi W, Hamzaoui A and Hamzaoui K: Interleukin-37 expression is decreased in Behçet's disease and is associated with inflammation. Immunol Lett 167: 87-94, 2015.

33. Charrad R, Berraïes A, Hamdi B, Ammar J, Hamzaoui K and Hamzaoui A: Anti-inflammatory activity of IL-37 in asthmatic children: Correlation with inflammatory cytokines TNF- $\alpha$, IL- $\beta$, IL-6 and IL-17A. Immunobiology 221: 182-187, 2016.

34. YeZ, Wang C, Tang J,Zhou Y, Bai L, Liu Y, Kijlstra A and Yang P. Decreased interleukin-37 expression in Vogt-Koyanagi-Harada disease and upregulation following immunosuppressive treatment. J Interferon Cytokine Res 35: 265-272, 2015.

35. Liu W, Deng L, Chen Y, Sun C, Wang J, Zhou L, Li H and Luo R: Anti-inflammatory effect of IL-37b in children with allergic rhinitis. Mediators Inflamm 2014: 746846, 2014.

36. Sharma S, Kulk N, Nold MF, Gräf R, Kim SH, Reinhardt D, Dinarello CA and Bufler P: The IL-1 family member 7b translocates to the nucleus and down-regulates proinflammatory cytokines. J Immunol 180: 5477-5482, 2008. 